\title{
Inteligencia versus asistencia a clases: su incidencia en el rendimiento académico
}

Rogers Eduardo Cabrera Sandoval ${ }^{1}$ Johanna Merari Moncada Ramos ${ }^{2}$

\section{RESUMEN}

El objetivo principal del estudio era analizar las relaciones existentes entre la inteligencia general y la asistencia a clases como predictores del rendimiento académico, en una muestra de estudiantes universitarios de distintas carreras. Los participantes fueron 56 alumnos matriculados en la clase de Psicología General, con un rango de edad de entre 18 y 35 años, de la Universidad Nacional Autónoma de Honduras, durante el tercer periodo académico de 2014.

El estudio tiene un enfoque cuantitativo, con un diseño transeccional correlacional. Para evaluar la capacidad intelectual $(\mathrm{Cl})$ se utilizó el test no verbal de la Universidad de Purdue; la asistencia a clases se determinó a partir de la presencia en el salón de clases y para definir el rendimiento académico se consideró como indicador principal el promedio de las notas correspondientes al primer y segundo parcial.

Los resultados revelan que no existe relación entre la inteligencia y el rendimiento académico $(r=-0.08$, Sig. $=0.553)$, mientras que la asistencia a clases sí se relaciona con este $(r=0.486$, Sig. $=0.000)$; por tanto, el análisis de correlación pone de manifiesto que la asistencia regular a clases está mucho más vinculada al éxito académico. Estos resultados incentivan el estudio de otras variables que pueden estar relacionadas con el rendimiento académico, de modo que otras investigaciones pueden abrir las puertas al mejoramiento del rendimiento académico estudiantil.

Palabras clave: inteligencia, asistencia a clases, rendimiento académico.

${ }^{1}$ Estudiante de la Carrera de Psicología, Facultad de Ciencias Sociales, UNAH: rogers.ps@outlook.

${ }^{2}$ Asesora, jefa del Departamento de Seguimiento y Control de la DSA, UNAH: johanna.moncada@ unah.edu.hn 


\section{ABSTRACT}

The main objective of this study was to analyze the relationships between general intelligence and school attendance as predictors of academic performance. In the study, a sample of 56 university students from different races was taken between age range of 18 and 35 years. All of the students enrolled in the class of General Psychology, during the third academic period of 2014 in the National Autonomous University of Honduras.

The present study has a quantitative approach with a correlational and transectional design. To evaluate the intellectual capacity of the students (IQ), a nonverbal test from Purdue University was used. The student attendance was verified from the presence in the classroom and in order to determine their academic performance, the average grades for the first and second periods were considered as the main indicator.

The results show that there is no relationship between intelligence and academic performance $(r=-0.08$, Sig. $=0.553)$ while attending classes is related $(r=0.486$, Sig. $=0.000$ ), therefore the correlation analysis shows that regular attendance is linked to academic success. These results encourage the study of other variables that can be linked to academic performance so that further research can identify how student achievement can be improved.

Keywords: intelligence, attendance, academic performance. 


\section{INTRODUCCIÓN}

La educación es un tema prioritario dentro de las políticas de todo país con aspiraciones de crecimiento y desarrollo, pero en muchas ocasiones el rendimiento estudiantil no cumple con las expectativas, esto sucede en todos los sectores del nivel educativo, independientemente de que sea público o privado. Por tanto, es de vital importancia el estudio de factores que determinen el rendimiento académico, para generar y promover propuestas sólidas y con bases científicas, propias de cada contexto educativo.

Probablemente una de las dimensiones más importantes en el proceso de enseñanza aprendizaje, tal como se ha venido definiendo hasta el momento, lo constituye el rendimiento académico del alumno. Cuando se trata de evaluar el rendimiento académico y cómo mejorarlo, se analizan en mayor o menor grado los factores que pueden influir en él (Benítez, Giménez y Osicka, 2000).

A esto se agrega el planteamiento de Jiménez (2000), quien expone que se puede tener una buena capacidad intelectual como también buenas aptitudes, sin embargo, no estar obteniendo resultados académicos (rendimiento) óptimos, ante la disyuntiva manifiesta de que el rendimiento académico es un fenómeno multifactorial y así debe realizarse su abordaje.

En tal sentido, es importante resaltar que se debe indagar y conocer los diversos factores que inciden en el rendimiento, en esto radica la importancia de conocerlos y describir qué elementos o condicionantes (por ejemplo: la asistencia a clases y la inteligencia) están relacionados con el nivel de aprovechamiento escolar, reflejado por medio de las notas de los alumnos.

La inteligencia es una de las variables más estudiadas dentro de los determinantes de índole personal. Históricamente, es un buen predictor de los resultados académicos que sobresale en el rendimiento académico, lo cual produce una relación significativa entre inteligencia y rendimiento académico; sin embargo, los coeficientes de correlación son moderados, lo que podría asociarse con la influencia recibida de variables como las sociales e institucionales (Castejón y Pérez, 1998).

En lo que a inteligencia se refiere, es importante identificar el tipo de inteligencia que se desee valorar como la emocional o la social, por ejemplo, y seleccionar adecuadamente sus metodologías evaluativas. Sobre la inteligencia emocional, en un estudio 
realizado por Montero y Villalobos (2004) con estudiantes universitarios en Costa Rica, se indica que el puntaje en la escala de inteligencia emocional en contextos académicos y el promedio de admisión a la universidad, presentan importantes niveles de asociación.

Basándose en lo anterior, resulta oportuno mencionar que muchos autores destacan la importancia del éxito escolar, de acuerdo con la percepción de Redondo (1997):

Requiere de un alto grado de adhesión a los fines, los medios y los valores de la institución educativa, que probablemente no todos los estudiantes presentan. Aunque no faltan los que aceptan incondicionalmente el proyecto de vida que les ofrece la institución, es posible que un sector lo rechace y otro, tal vez el más sustancial, solo se identifica con el mismo de manera circunstancial (p.54).

González-Pienda (2003) añade que:

Aunque el binomio éxito-fracaso hace referencia a una normativa general sin tener en cuenta, a veces, el proceso evolutivo y las diferencias individuales de cada alumno, lo cierto es que, en muchos casos, la situación de bajo rendimiento o fracaso lleva consigo una serie de problemas y tensiones emocionales que repercuten en el desarrollo personal e, incluso, pueden llevar a una deficiente integración social. Al hablar de fracaso hay que tener en cuenta que no estamos hablando de estudiantes torpes, sino de alumnos inteligentes que no rinden o que no logran el rendimiento deseado dentro del tiempo estipulado y, consecuentemente, aparecen como malos estudiantes (p. 247).

Unas veces se trata de una situación poco duradera y transitoria. Otras veces, en cambio, es permanente y perturbadora. El fracaso también se puede referir a la propia institución escolar y, de hecho, muchas investigaciones en vez de plantearse por qué tantos fracasan, empiezan a cuestionar por qué las escuelas están fracasando (González - Pineda y Núñez, 2002). Bajo esta misma línea González Pineda (2003) expresa lo siguiente:

A la hora de delimitar qué factores están incidiendo en el éxito o fracaso escolar, no es extraño encontrarse con muchas dificultades, debido a que dichos factores 0 variables constituyen una red fuertemente entretejida, por lo que resulta difícil delimitarlas para atribuir efectos claramente discernibles a cada una de ellas. En los últimos años, se ha dado un avance muy relevante en el sentido de superar los enfoques clásicos con una finalidad predictiva, pasando a otros más complejos con una finalidad explicativa a través de modelos estructurales o causales. Al conjunto de variables que indicen en el éxito o fracaso se les conoce como condicionantes del rendimiento académico (p. 247). 
Específicamente en el campo de la inteligencia conviene resaltar que el uso de los test de inteligencia proviene de la fuerte asociación entre cognición y aprendizaje, es decir, entre aptitudes cognitivas y rendimiento escolar. Si esto es así, los test de inteligencia ayudan en la consecución de objetivos de diagnóstico y de pronóstico de las potencialidades y dificultades de los alumnos en sus aprendizajes y desempeños académicos (Barca y Peralbo, 2002).

En particular, los test de factor "g" y las escalas de $\mathrm{Cl}$ son, actualmente, considerados los mejores predictores simples del desempeño académico. Bajo esta línea teórica existen diversas líneas de investigación sobre la relación entre cognición y aprendizaje -y es conveniente asumir esta relación como recíproca-, lo cual ha revelado que en los coeficientes de correlación obtenidos no se presentan constantes a lo largo de toda la escolarización y oscilan en función de las áreas curriculares y de las propias aptitudes cognitivas que se consideren en los estudios (González Pineda, 2003).

Al mencionar la variable inteligencia con relación al rendimiento académico, cabe destacar la investigación reciente de Pizarro y Crespo (2000) sobre inteligencias múltiples y aprendizajes escolares, en donde expresan que la inteligencia humana no es una realidad fácilmente identificable, es un constructo utilizado para estimar, explicar o evaluar algunas diferencias conductuales entre las personas: éxitos y fracasos académicos, modos de relacionarse con los demás, proyecciones de proyectos de vida, desarrollo de talentos, notas educativas, resultados de test cognitivos, etc. Los científicos, empero, no han podido ponerse muy de acuerdo respecto a qué denominar una conducta inteligente.

Por tanto, resulta importante considerar otro tipo de variables al margen de las calificaciones y el nivel de inteligencia de los estudiantes, las que aparentemente inciden en el rendimiento académico y que valdría la pena mencionar.

Es importante destacar que la asistencia escolar tiene un gran impacto en el éxito académico de un estudiante. Aun cuando los niños crecen y se vuelven más independientes, las familias siguen jugando un rol clave en asegurarse que asistan diariamente a la escuela, colegio e inclusive a la universidad, sobresaliendo de esta forma que la asistencia es considerada importante para el éxito en todos los niveles educativos, lo cual probablemente es considerado como un signo de responsabilidad que también se verá reflejado en un futuro dentro del campo laboral.

En el trabajo de investigación realizado por Pérez, Ramón y Sánchez (2000) con estudiantes universitarios, se encontró que la motivación está asociada a la 
asistencia a clases y que la ausencia al salón de clases se relaciona con problemas de repetición y abandono de los estudios. Asimismo, a mayor asistencia, mejor calificación; la asistencia es una de las variables más significativas que influye en el rendimiento académico del alumno.

En este estudio se abordarán dos determinantes de índole personal: la inteligencia general comparada con la asistencia a clases, a fin de establecer cuál es la relación de estos factores de forma individual con el rendimiento académico y determinar el peso que poseen tales variables en el estudiantado universitario.

\section{METODOLOGÍA}

\section{Diseño}

El presente estudio tiene un enfoque cuantitativo, con un diseño transeccional correlacional (no experimental), en donde se busca establecer si existe una relación en factores como la inteligencia y la asistencia a clases, con respecto al rendimiento académico en estudiantes universitarios.

\section{Participantes}

La población de esta investigación son alumnos de la clase de Psicología General (sección 1500) de la Universidad Nacional Autónoma de Honduras (UNAH), de ciudad universitaria, durante el tercer periodo académico de 2014. Se utilizó un muestreo no probabilístico, contando con la participación voluntaria de 56 estudiantes, los cuales tienen edades que oscilan entre los 18 y 32 años, los mismos pertenecen a diversas carreras y años de estudio.

Intervenciones

Se utilizó el test no verbal de la Universidad de Purdue para determinar la capacidad intelectual de los participantes. La prueba mide razonamiento abstracto y consta de 48 ítems, cuyo nivel de dificultad se incrementa al irse desarrollando la misma. Para determinar el rendimiento académico se tomaron las notas correspondientes al primer y segundo parcial. También se aplicó un cuestionario de preguntas donde el 
sujeto debe especificar sus datos de generales (edad, sexo, estado civil, año de ingreso a la universidad, número de clases aprobadas, carrera, ingreso económico familiar, creencia religiosa, ideología política, si es activista político y si pertenece a una organización política).

\section{Análisis estadístico}

Para realizar los análisis correspondientes se usó el software IBM SPSS Statistics 21. Se utilizó la correlación no paramétrica de Rho de Spearman para comprobar la existencia o no de una relación entre la asistencia a clases, la inteligencia y el rendimiento académico.

\section{ANÁLISIS E INTERPRETACIÓN DE RESULTADOS}

En los resultados siguientes se hace un análisis de los factores asociados al rendimiento académico en estudiantes universitarios.

Inteligencia, asistencia y rendimiento académico

La inteligencia se considera como un elemento clave para el éxito, de aquí estriba el interés por ver qué relación presenta esta con el rendimiento académico, pero también se insiste bastante por los diversos agentes del sistema educativo en la asistencia regular a clases. Por ello, a continuación se detalla la correlación entre las variables mencionadas.

El coeficiente intelectual del estudiantado en promedio es de 95 , lo cual es considerado normal. De igual manera, de la capacidad intelectual se desprenden diversos rangos o clasificaciones, distinguiendo así entre el nivel intelectual del estudiantado, pero a pesar de tal jerarquización esta no denota visiblemente diferencias significati- 
Tabla 1. Clasificación de la capacidad intelectual y el rendimiento académico de los estudiantes universitarios

\begin{tabular}{lll}
\hline Capacidad intelectual & Frecuencia & Nota promedio \\
\hline Limítrofe & 6 & 68,00 \\
\hline Normal bajo & 12 & 59,58 \\
Normal & 32 & 66,19 \\
Normal alto & 6 & 59,67 \\
Total & 56 & 64,27
\end{tabular}

Con relación al porcentaje de asistencia, se observa que a medida que la asistencia mejora, también se incrementan las notas en promedio (rendimiento) del estudiantado (ver tabla 2).

Tabla 2. Asistencia y rendimiento académico de los estudiantes universitarios

\begin{tabular}{|lll|}
\hline Asistencia & Frecuencia & Nota promedio \\
\hline Baja & 12 & 50,33 \\
\hline Regular & 25 & 64,84 \\
Alta & 19 & 72,32 \\
\hline Total & 56 & 64,27 \\
\hline
\end{tabular}

Por tanto, en niveles absolutos no existe relación entre la inteligencia y el rendimiento académico en el estudiantado que participó en el estudio $(r=-0.08$, Sig. $=0.553)$. En contraste, se encontró que sí hay una relación entre la asistencia a clases y el rendimiento académico $\left(r=0.486^{\star *}\right.$, Sig. $\left.=0.000\right)$, lo que indica que en la medida que los estudiantes asisten con mayor frecuencia a clases, estos tendrán un mayor rendimiento académico.

\section{DISCUSIÓN Y CONCLUSIONES}

Los resultados obtenidos acerca de las relaciones entre la inteligencia y la asistencia a clases con el rendimiento muestran ser distintas, ya que la inteligencia no refleja ningún tipo de relación, mientras que con la asistencia se encontró una correlación positiva muy significativa, lo cual indica que es más importante hacer acto de presencia en el aula de clases, más allá de solo depender de las capacidades individuales para aprender. 
Estos resultados son un aporte inicial que brinda información sobre la importancia de ciertos elementos para generar un mejor rendimiento académico. Investigaciones de este tipo sirven de punto de partida para las incorporaciones de otros condicionantes, de forma que permitan describir de forma más clara y precisa las mejores condiciones para estimular el crecimiento individual del estudiantado, en este caso el universitario.

No obstante, hay que ser cuidadosos con tales resultados, puesto que sería adecuado buscar la generalización e incorporar procesos más amplios, rigurosos y precisos que permitan encontrar más determinantes del rendimiento académico que promuevan de esta manera el desarrollo del estudiantado universitario, en aras de su preparación para el campo laboral, para que estos puedan sacar el máximo provecho de su aprendizaje y de esta forma puedan ser mejores profesionales.

El debate sobre la inteligencia y la asistencia a clases debe continuar siendo explorado, pero se ve reflejada que la presencia en el salón de clases es vital, esto incentiva a realizar otros estudios que permitan adicionar nuevos elementos para analizar la atención, la disposición para aprender e inclusive la inteligencia emocional, para promover de esta manera un desarrollo académico con calidad en el estudiantado.

Como reflexión final, se destaca que la educación es la llave del éxito, pero que la mayor riqueza está en quien utiliza lo aprendido para generar cambios positivos en una sociedad que cada día más adolece de sueños y esperanzas.

\section{AGRADECIMIENTO}

A los estudiantes de la clase de Psicología General, sección 1500, del tercer periodo académico de 2014 de la UNAH, que voluntariamente se ofrecieron para ser parte del estudio. 


\section{REFERENCIAS}

Barca, A. y Peralbo, M. (2002). Los contextos de aprendizaje y desarrollo en la educación secundaria obligatoria (ESO). Perspectivas de intervención psicoeducativa sobre el fracaso escolar en la comunidad autónoma de Galicia. Informe final del Proyecto FEDER (1FD97-0283). Madrid: Ministerio de Ciencia y Tecnología.

Benítez, M; Giménez, M. y Osicka, R. (2000). Las asignaturas pendientes y el rendimiento académico: ¿existe alguna relación? Recuperado de: http//fai.unne.edu.ar/links/LAS\% 2...20EL\%20RENDIMIENTO\%20ACADEMICO.htm

Castejón, J. L. y Pérez, A. M. (1998). Un modelo causal-explicativo sobre la influencia de las variables psicosociales en el rendimiento académico. Bordón, 50, 171-185.

González Pineda, J. A. (2003). El rendimiento escolar. Un análisis de variables que lo condicionan. Revista Galego-Portuguesa de Psicoloxía e Educación, 8, 247.

González Pineda, J. A. y Núñez, J.E. (2002). Manual de psicología de la educación. Madrid: Pirámide.

Jiménez, M. (2000). Competencia social: intervención preventiva en la escuela. Infancia y Sociedad, 24, 21-48.

Montero Rojas, Eilena y Villalobos Palma, Jeannette. (2004). Factores institucionales, pedagógicos, psicosociales y sociodemográficos asociados al rendimiento académico y a la repetición estudiantil en la Universidad de Costa Rica. Instituto de Investigaciones Psicológicas, Universidad de Costa Rica.

Pérez Luño, A.; Ramón Jerónimo, J. y Sánchez Vázquez, J. (2000). Análisis exploratorio de las variables que condicionan el rendimiento académico. Sevilla: Universidad Pablo de Olavide.

Pizarro, R. y Crespo, N. (2000). Inteligencias múltiples y aprendizajes escolares. Recuperado de: http: //www.uniacc.cl/ talon/ anteriores/talonaquiles5/tal5-1.htm

Redondo, R. J. (1997). La dinámica escolar: de la diferencia a la desigualdad. Revista de Psicología, 6, 54. Chile. 